\title{
Segregated the Protoscolices of Hydatid Cysts Parasite by Medical Image Processing
}

\author{
Kawther H. Al-khafaji \\ Kufa University, College of Education for Girls, Physics Department, Iraq
}

\begin{abstract}
Hydatid disease is an important zoonotic parasitic disease with public health significance that affects both humans and other mammals such as sheep, dogs, rodents and horses, currently the surgery is the best way for treatment in hydatid Cysts, a major concern during surgery to remove cysts is that brood capsules can float free within the cyst and that lead to spillage of viable parasite (Protoscolices), Therefore the surgeons inject the cyst by Scolicidal agent before removing the cyst and that lead to killing most or in some time all the Protoscolices in the cyst. The aim of current research is to produce a an effective image processing program that can detect and separate the Protoscolices of hydatid cysts dead from live in the microscopic medical image, depending on color detection technique by using Matlab image processing function and tools.The results indicate that the algorithm proposed are able to isolate the dead Protoscolices in hydatid cysts microscopic images.
\end{abstract}

Keywords: Image processing, Matlab, Hydatid Cysts, Protoscolices, Scolicidal, Segregation, Colors Detection

\section{Introduction}

Medical Imaging is one of the most important application areas of digital image processing, in today's health care, medical imaging plays an important role throughout the entire clinical process from diagnostics and monitoring the patient's health condition and providing an effective treatment and surgical procedures and follow up studies[1]. The influence and impact of digital images on modern society is tremendous, and image processing is now a critical component in science and technology. The rapid and continuing progress in computerized medical image reconstruction, and the associated developments in analysis methods and computer-aided diagnosis, have propelled medical imaging into one of the most important subfields in scientific imaging [2].

The last two decades have witnessed significant advances in medical imaging and computerized medical image processing. These advances have led to new two, three, and multidimensional imaging modalities that have become important clinical tools in diagnostic radiology [3].

Medical Image Processing brought a great contribution in visualization of human anatomy and radically enhanced the Computer-aided diagnostic systems. It assists medical practitioners for detection and localization of pathological deformations. In which, the advanced Digital Image Processing techniques are used to analyze the various internal structures of body of the patients [4].

Advanced techniques of image processing and analysis find widespread use in medicine. In medical applications, image data are used to gather details regarding the process of patient imaging whether it is a disease process or a physiological process. Information provided by medical images has become a vital part of today's patient care. The images generated in medical applications are complex and vary notably from application to application[5].

\section{Hydatid Disease}

Hydatid disease or Echinococcal disease, is a parasitic disease that affects both humans and other mammals such as sheep, dogs, rodents and horses. There are three different forms of Echinococcosis found in humans, each of which is caused by the larval stages of different species of the tapeworm of genus Echinococcus[6]. tapeworm eggs are passed with the feces of infected carnivores and may subsequently infect humans who inadvertently ingest them. Clinical manifestation of hydatidosis is characterized by tumor like growths that occur mostly in the liver and lungs, with varying degrees of infestation of other organs. These growths are usually filled with a watery fluid known as 'hydatid cyst fluid.' Hydatidosis is a major world zoonosis affecting humans as well as domestic animals .Because of the slow progression of the disease, it may initially be asymptomatic or show very slight manifestations. However, serious clinical symptoms may eventually develop, which vary, depending on the extent of infestation, the site of infection, and the size of the cyst As with other parasitic diseases [7]. Human infection may occur after ingestion of infective eggs passed in the feces from dogs through direct contact or via environmental contamination. The definitive host becomes infected by ingesting Protoscolices produced by asexual multiplication of the metacestode (Mesocestoides).There may be several thousand Protoscolices within a single cyst, and each one is capable of developing into a sexually mature adult worm [8]. The disease is endemic in the Mediterranean area, in the Middle East, the Baltic areas, South America, India, Northern China and other sheep raising areas, however, considering the increased travels and tourism all over the world, it can be found anywhere, even in developed countries [9]. Although most hydatid cysts are found in the liver and lung, the disease can arise anywhere in the body [8].

\section{Hydatid Cyst Scolicidal}

Surgical removal of the intact hydatid cyst is the most preferred method of therapy . one of the major surgical complications of hydatidosis is recurring (secondary) cystic 


\section{International Journal of Science and Research (IJSR) \\ ISSN (Online): 2319-7064}

Index Copernicus Value (2013): 6.14 | Impact Factor (2014): 5.611

echinococcosis after operation for primary hydatid disease. Dissemination of protoscolexrich fluid during surgery is a major cause of recurrence and multiple secondary echinococcosis [8].

Use of an effective Scolicidal (protoscolicidal) adjunct in hydatid surgery is an important procedure which may reduce the recurrence rate, Therefore, the surgeons inject the cyst by Scolicidal agent like alcohol or Formalin,.... etc, and all those procedure are applied before removing the cyst and that lead to killing most or some time all the Protoscolices in the cyst depending on the Scolicidal agent sort. After inject process, the surgeons found there is no different in appearance between live and dead Protoscolices, figure(1) shown live Protoscolices, hence they are use eosin stain with very small concentration (1 gram of eosin powder in 1000 $\mathrm{mL}$ of distilled water) for the viability test of Protoscolices. After few minute of exposure, the Protoscolices that did not take the dye in, accepted as potentially viable and those done, were considered dead as we shown in figure(2). figure(3) display live and dead Protoscolices together in the same sample, all those images are taken under light microscopy.

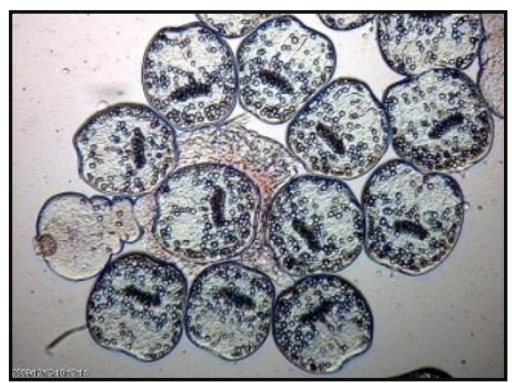

Figure 1: Live Protoscolices [6]

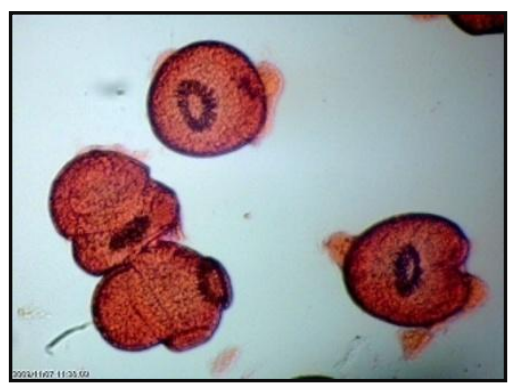

Figure 2: Dead Protoscolices [6]

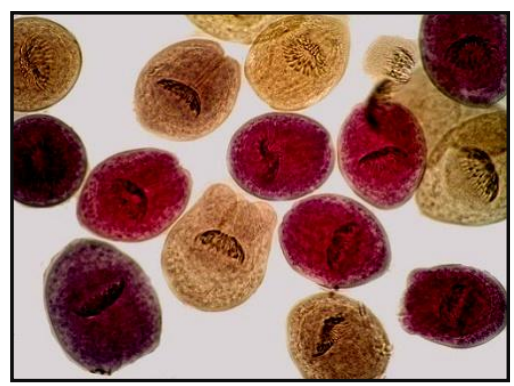

Figure 3: Live and Dead Protoscolices

\section{Color Detection}

The human visual system can distinguish hundreds of thousands of different color shades and intensities, but only around 100 shades of grey. Therefore, in an image, a great deal of extra information may be contained in the color, and this extra information can then be used to simplify image analysis, e.g. object identification and extraction based on color. Three independent quantities are used to describe any particular color. The hue is determined by the dominant wavelength. Visible colors occur between about $400 \mathrm{~nm}$ (violet) and $700 \mathrm{~nm}$ (red) on the electromagnetic spectrum.

The saturation is determined by the excitation purity, and depends on the amount of white light mixed with the hue. A pure hue is fully saturated, i.e. no white light mixed in. Hue and saturation together determine the chromaticity for a given color. Finally, the intensity is determined by the actual amount of light, with more light corresponding to more intense colors [10].

Color information is useful in vision based feature detection ,often characteristic color detection is applied as a preprocessor followed by a shape matching algorithm to identify the target feature. Among them, characteristic color detection has been an attractive solution particularly in applications where the color difference between the target features and its background is significant [11]. The objects may be further evaluated for their colors. This is done by averaging the RGB content within the boundaries of the objects. For example an object with a relatively high average value of $\mathrm{R}$ over its surface, may possess a shade of red [12].

In this research we present an color detection algorithm to find objects in an microscopic medical image that have a specific color (red), in the same time we differentiate this objects from another objects.

\section{MATLAB}

In recent years, Matlab, the product of MathWorks, has become a popular tool for fast development. Its many Toolboxes, powerful interface and user friendliness make it a tool of choice in many disciplines, including medical image processing [13]. The toolbox functions implemented in the open MATLAB language can be used to develop the customized algorithms [14]. Matlab allows matrix computation, implementation of algorithms, simulation, plotting of functions and data, signal and image processing by the Image Processing Toolbox [5].

Image Processing Toolbox in MATLAB provides a comprehensive set of reference-standard algorithms and graphical tools for image processing, analysis, visualization, and algorithm development. User can perform image enhancement, feature detection, noise reduction, image segmentation, geometric transformations, and image registration without serious difficulties [2].

\section{Research Plan}

In this research we presented an algorithm to separate the Protoscolices of Hydatid Cysts Parasite in microscopic 


\section{International Journal of Science and Research (IJSR) ISSN (Online): 2319-7064 \\ Index Copernicus Value (2013): 6.14 | Impact Factor (2014): 5.611}

images Based on color detection techniques due to the fact that is the color of dead Protoscolices become red after it coloring by eosin stain, our concern concentrate on development the color detection techniques and that lead to develop an particular algorithm, we used MATLAB (R2012b) software to implement the algorithm .we obtained an visual result after applied the particular algorithm on medical images.

\section{Design and Implementation}

To separate the Protoscoleces of hydatid cyst disease (the dead from live) in medical images we have proposed the following algorithm:-

\section{Step1: Image Acquisition}

The image acquisition is done using a medical image as an sample image where we used imread function in this step.

Step2: Display the original image

using imshow function to display the image .

Step3: Split Image into three color bands

In this step we separate the medical image (sample image) into three color bands(red, green ,blue)

\section{Step4: Threshold each color band}

Step5: combine the all Threshold color band

Step6: fill image regions and holes

we fill image regions and holes by using imfill function, and this include fills holes in the binary image, a hole is a set of background pixels that cannot be reached by filling in the background from the edge of the image.

Step7: convert fill image in to 8-bit unsigned integer

we us uint 8 function to convert the fill image in to 8bit unsigned integer, which mean converts the elements of an array into unsigned 8-bit (1-byte) integers of class uint8.

Step8: Detach the Protoscolices of Hydatid Cysts

We used imadjust function to Adjust image intensity values, and this increases the contrast of the output image and lead to Separate the dead Protoscolices from live Protoscolices.

Figure(4) shows the flowchart of the suggested algorithm

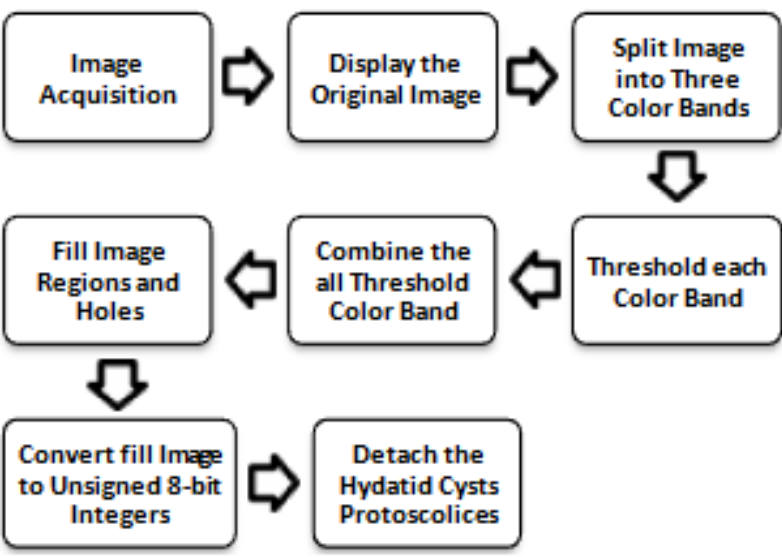

Figure 4: Flow chart of the proposed methodology

\section{Experimental Results and Discussion}

As we know onetime the surgical removal of hydatid cyst is the most method of therapy are usually used, but the flood of hydatid cysts content into the human body organs is one of most riskiness in this process, therefore we use the Scolicidal before the surgery, that cause to killed the most Protoscolices in cysts, the aim of this research is to find objects of a certain color, In this experimental work we have taken an microscopic medical image. The field of microscopic images contain two sort of Protoscolices, the dead Protoscolices have red color and other Protoscolices in the same field have an variant color, because of that we had applied the proposed algorithm depending on colors detection technique to separate those objects and we eliminating unwanted color while execution of algorithm , therefore we neglected the live Protoscolices from the image field and in same time we leave the dead Protoscolices, the result show the image with only the requested Protoscolices showing and everything else blacked out, that lead up to reduce time identification and decrease indistinctness of the vision system, and subsequently it give as an assessment to succeed of Scolicidal in killing the Protoscolices. This experimental work is carried out in Matlab R2012b, The working scheme is showed in figure (5).

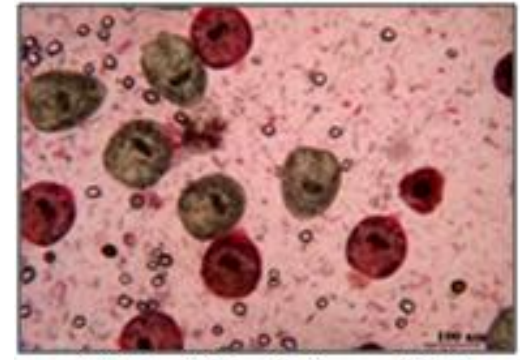

(a) The Original Image [15]

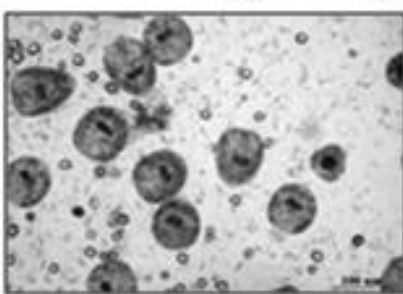

(b) RedBand

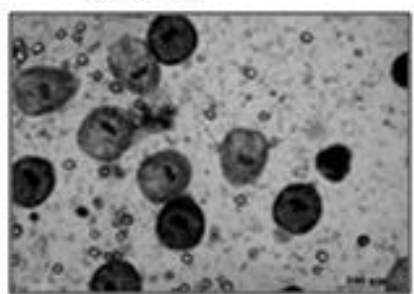

(c) Green Band

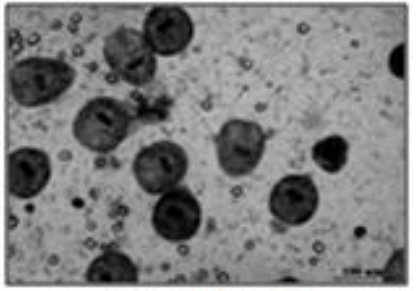

(d)Blue Band

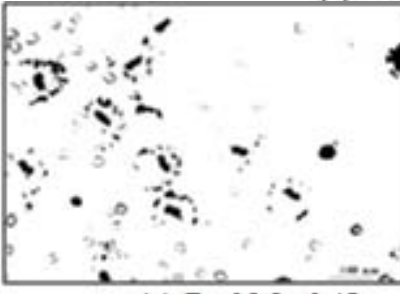

(e) Red Mask(f)

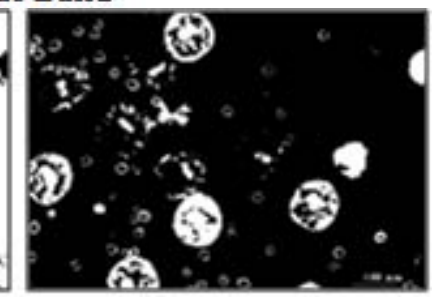

(f) Green Mask 


\section{International Journal of Science and Research (IJSR) \\ ISSN (Online): 2319-7064}

Index Copernicus Value (2013): 6.14 | Impact Factor (2014): 5.611

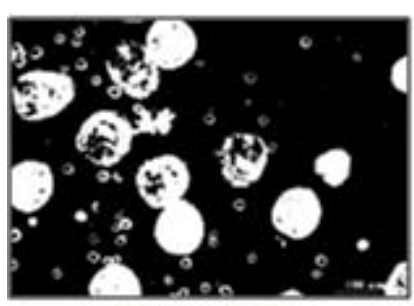

(g)Blue Mask

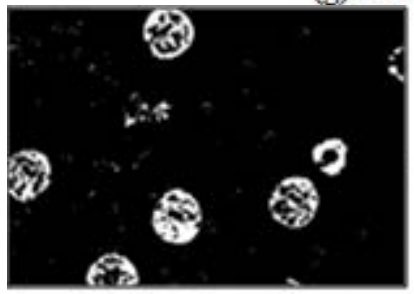

(h) Combined Mask

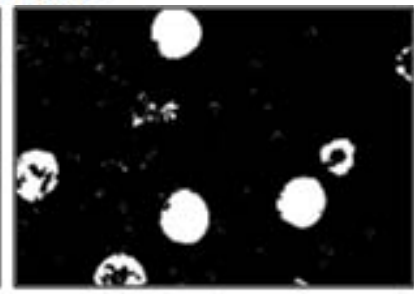

(i) Filled Mask

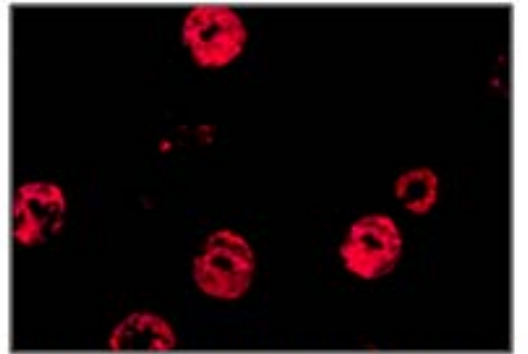

(j) Red Object only (Live Protoscolices)

Figure 5: The working scheme of Segregate algorithm

\section{Conclusion}

In this paper, the algorithm software presented an accurate result in segregate process, The principle of research work is using color detection technique to detection and separation the Protoscolices of hydatid cysts in microscopic medical images, This provides a diagnosis and preoperative planning surgery significant information (how many Protoscolices are killed and how many are still alive ,before the Hydatid Cysts contain is flooded) and this is very important in hydatid treatments, because it gives a thought to scolicidal agent qualifications, The results of the image can be used as good input in determining the dead Protoscolices, Further studies will be focused on complete Protoscolices count i.e. a total count of the number of dead Protoscolices, live Protoscolices .This can be easily done by modifying the present software to take into account their different color.

\section{References}

[1] I. Scholl ,T., Aach · T. M., Deserno - T., Kuhlen , "Challenges of medical image processing," SpringerVerlag ,Compute Sci Res Dev, Special Issue Paper 26,pp 5-13,2011.

[2] G. Dougherty ,Digital Image Processing for Medical Applications, California State University, Cambridge University Press, 2009.

[3] A. P. Dhawan, Medical Image Analysis, Second Edition, Institute of Electrical and Electronics Engineers, Copyright 2011.

[4] M. Y.Khachane, R. J Ramteke, "Fuzzy Rule based Multimodal Medical Image Edge Detection," International Journal of Computer Applications (0975 -
8887) Recent Advances in Information Technology, India, 2014.

[5] M.Lyra, A.Ploussi , A.Georgantzoglou, "MATLAB as a Tool in Nuclear Medicine Image Processing," Radiation Physics Unit, A Radiology Department, University of Athens Greece, MATLAB - A Ubiquitous Tool for the Practical Engineer ,pp. $477-$ 500,2011

[6] A.N. Assi "Effectivness of $10 \%$ povidone iodine as ascolicidal agent in hydatid cyst surgery in compared with other scolicidal agents ," Qatar Medical Journal, VOL.9 No.16, 2013.

[7] A. K. Barzinji, , R. A. Mothana, A. K. Nasher, “ Effect of leaf extracts of Dendrosicyos socotrana and Jatropha unicostata on the viability of Echinococcus granulosus protoscoleces, " EurAsian Journal of BioSciences, 122-129, DOI:10.5053, Yemen, 2009.

[8] M. Moazeni, A.Roozitalab, “ High scolicidal effect of Zataria multiflora on protoccoleces of hydatid cyst: an in vitro study, " Springer-Verlag London Limited ,Comp Clin Pathol 21:pp.99-104,2010.

[9] M. Moazeni, A. Nazer, “ In vitro lethal effect of Zingiber officinale R. on protoscolices of hydatid cyst from sheep liver, " Microbiology Research, Shiraz University, volume 2:e25, Iran ,2011

[10] R.C. Gonzales,Woods R.E., Digital Image Processing, Addison-Wesley, Reading, 1992.

[11] K. Lee, , Q. Li, W. Daley , “ Effects of Classification Methods on Color-Based Feature Detection With Food Processing Applications, " IEEE Transactions on Automation Science and Engineering, VOL. 4, NO. 1, 2007.

[12] S.Rege, R.Memane, M.Phatak, and P.Agarwal, “ 2D Geometric Shape and Color Recognition Using Digital Image Processing," International Journal of Advanced Research in Electrical, Electronics and Instrumentation Engineering Vol. 2, Issue 6,pp.2479 -2487, June 2013.

[13] M.Bister, C.S.Yap, K.H.Ng, C.H. Tok, "Increasing the speed of medical image processing in Matlab, Biomedical Imaging and Intervention Journal, 2007.

[14] E.D.SELEȚCHI, “ Medical Image Processing using MATLAB ", University of Bucharest, Romania,2008.

[15] D. Yones, G. Taher, Z .Ibraheim , "In vitro effects of some herbs used in Egyptian traditional medicine on viability of protoscolices of hydatid cysts, " openi.nlm.nih.gov, - Korean J. Parasitol, 2011. [Online]. Available: http://openi.nlm.nih.gov/detailedresult.php?img=32108 42_kjp-49-255-g005\&req=4. [Accessed: octoper. 17, 2014]

\section{Author Profile}

Kawther H. Al-khafaji received the B.S. degrees in Physics Science in 2005 from Tikrit University, College of Education, and M.S. degrees in Image Processing from Kufa University, College of Education for girls in 2012, during 2006 she working in Physics Department/ College of Education for girls and she is still working so far in the same mentioned college. 